\title{
Phospholipid Polymer Multilayered Hydrogels Containing Cells for Cancer Drug Screening
}

\author{
Botao Gao $^{1 *}$, Tomohiro Konno ${ }^{2}$ and Kazuhiko Ishihara ${ }^{1,2}$ \\ 1 Department of Materials Engineering, 2 Department of Bioengineering, School of Engineering, \\ The University of Tokyo, 7-3-1, Hongo, Bunkyo-ku, Tokyo 113-8656, Japan \\ * Corresponding author: e-mail: gao@mpc.t.u-tokyo.ac.jp
}

\begin{abstract}
Hydrogel multilayers with micrometer-scale in thickness containing living cancer cells were prepared by layer-by-layer assembling with 2-methacryloyloxyethyl phosphorylcholine polymer solution and poly(vinyl alcohol) solution alternatively under biological conditions. It was demonstrated that this tool could be utilized for minimizing barriers to the diffusion of drugs while preserving the $3 \mathrm{D}$ context. The contribution of dimensional and diffusional factors on drug efficacy was also investigated. As a case study, the cytotoxicity effects of paclitaxel on human cervical cancer HeLa-Fucci cells were evaluated in vitro. A comparative study was carried out: 2D culture, 3D culture in microgel thin membrane and in macrogel. Time-lapse microscopic analyses showed HeLa-Fucci cells cultured on 2D exhibited a more rapid and robust response to stimulation than cells cultured in microgel thin membrane. Besides, HeLa-Fucci cells cultured under macrogel did not show any response to stimulation. It was speculated that both of the diffusion of paclitaxel and matrix dimensionality could affect drug efficacy and diffusion of drug seemed to play a more important role. This study provides a versatile and experimentally convenient method to fabricate a useful and generalizable platform for cancer drug screening.
\end{abstract}

Key words: Phospholipid polymer, layer-by-layer assembly, hydrogel membrane, 3D culture, diffusion

\section{INTRODUCTION}

The efficacy of anticancer drugs is usually tested with cells on two dimensional (2D) culture plates. However, these drugs do not work as effectively in vivo as in $2 \mathrm{D}$ cell culture. This discrepancy in drug efficacy, in part, could be due to the three-dimensional (3D) nature of tumor $v s$ the 2D nature of cell culture [1]. Therefore, it would be useful to develop a 3D in vitro model of tumor that would resemble a $3 \mathrm{D}$ tumor microenvironment to obtain a realistic assessment of drug efficacy prior to their testing in animal models or patients. However, other issue is that, at present, cells cultured within 3D matrices are typically embedded within millimeter-scale hydrogels (macrogels). Numerous studies have demonstrated that diffusion-mediated transport plays a key role in $3 \mathrm{D}$ matrices, resulting in spatiotemporal differences in concentrations of drug stimuli [2]. This asynchronous stimulation can obscure detection of drug efficacy. Consequently, whereas differences in drug efficacy are easily observed between 3D and 2D culture, differences induced by diffusional or dimensional factors have been substantially more difficult to confirm.

Here we set out to determine whether distinct drug response seen in cells cultured within 3D are entirely due to the $3 \mathrm{D}$ confinement or in part from delays in the diffusion of drugs to cells.

In order to realize this purpose, we prepared a micrometer-scale hydrogel membrane to minimize spatial and temporal gradients due to limitations in the transport of factors through 3D matrices, such that diffusible factors introduced to the medium would quickly equilibrate inside these ultrathin hydrogel membranes. This hydrogel membrane was prepared by the combination of the simple dropping/spinning-assisted layer-by-layer (LbL) procedure and polymers that can form hydrogels spontaneously $[3,4]$. We have reported that a water soluble 2-methacryloyloxyethyl phosphorylcholine (MPC) polymer [poly(MPC)-co- $n$-butyl methacrylate(BMA)-co-p-vinylphenylboronic acid(VPBA)](PMBV)] can crosslink with poly(vinyl alcohol) (PVA) and form hydrogel in aqueous medium [5]. This PMBV/PVA hydrogel system is particularly suitable for this study. The MPC units are well known for their cytocompatibility and high ability to suppress protein adsorption, cell adhesion and subsequent biological reactions, so cells do survive well in the PMBV/PVA hydrogel but without biochemical cell-matrix interactions and only physical cues remained, such as 3D confinement and diffusion. Therefore, this hydrogel creates a milieu to decouple physical cell-matrix interactions (3D confinement, diffusion) from biochemical cell-matrix interactions. By comparative drug efficacy study in micrometer scale hydrogel, millimeter scale hydrogel and 2D culture plates, we are able to decouple and distinguish the contribution of dimensional and diffusional factors on drug efficacy.

\section{MATERIALS AND METHODS}

\subsection{Materials}

MPC was obtained from NOF Corporation (Tokyo, Japan), which was synthesized by a previously reported method [6]. BMA was purchased from Kanto Chemicals (Tokyo, Japan), VPBA was purchased from Tokyo 
Chemical Industry (Tokyo, Japan), and PVA (mean polymerization degree:1,000, saponification degree:96.0 mol\%) was purchased from Wako Pure Chemical (Osaka, Japan). Other organic reagents and solvents were commercially available reagents of extra-pure grade and were used without further purification.

PMBV was synthesized by radical random copolymerization of corresponding monomers, as previously described [3-5]. The mole fractions of MPC, BMA, and VPBA units in the obtained PMBV were determined to be $0.60,0.25$, and 0.15 , respectively, using ${ }^{1}$ H-NMR (JNM-NR30; JEOL, Tokyo, Japan). The weight-averaged molecular weight of PMBV was $1.7 \times$ $10^{4}$, as determined by gel permeation chromatography (Jasco, Tokyo, Japan), with poly(ethylene oxide) standards. The chemical structure of PMBV and the gelation mechanism between PMBV and PVA are shown in Fig. 1(a) and (b), respectively. $5.0 \mathrm{wt} \%$ PMBV and $5.0 \mathrm{wt} \%$ PVA were prepared with PBS for gelation.

\section{(a) $\operatorname{PMBV}(a / b / c=0.60 / 0.25 / 0.15)$}

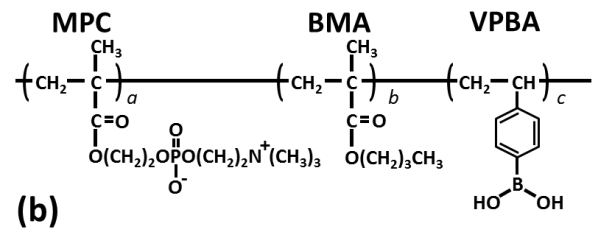

PMBV solution PMBV/PVA hydrogel

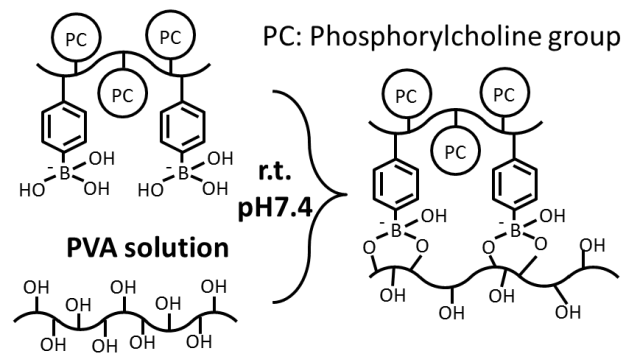

Fig. 1 (a) The chemical structure of PMBV, and (b) gelation mechanism between PMBV and PVA.

\subsection{Cell culture}

The model cells were human cervical cancer HeLa cells stably expressing fluorescent ubiquitination-based cell cycle indicator (Fucci) [7] fusion proteins as cell cycle sensors. Cell proliferation cycle goes from G1 to $\mathrm{M}$ phase through S, G2 and M phases. The Fucci technology allows dual-color imaging, which can distinguish between live cells in the G1 and the S/G2/M phases in the cell proliferation cycle. This technology allows for in vivo or in vitro analysis of spatial and temporal patterns of cell cycle dynamics, owing to the brightness of the fluorescence and the high contrast between the two colors (red and green). HeLa-Fucci cells were purchased from Riken Cell Bank (Ibaraki, Japan) and were cultured with DMEM (Sigma-Aldrich, St. Louis, MO) supplemented with $10 \%$ FBS. All cells were cultured at $37^{\circ} \mathrm{C}$ in a $5.0 \mathrm{vol} \% \mathrm{CO}_{2}$ humidified atmosphere.

2.3 Cell encapsulation into PMBV/PVA ultrathin

hydrogel membrane

HeLa-Fucci cells were dispersed in $150 \mu \mathrm{L}$ of the 5.0 wt $\%$ PMBV solution. The cell density of each cell suspension was $5.0 \times 10^{6}$ cells $/ \mathrm{mL}$. Briefly, the PMBV solution was manually spread onto a dish to form a thin membrane. Then $150 \mu \mathrm{L}$ of PVA solution was deposited onto this PMBV precursor layer. After a waiting period of $120 \mathrm{~s}$ to allow the reaction between VPBA units and the hydroxyl groups, the dish was rotated at $2000 \mathrm{rpm}$ to remove the excess PVA solution and form a uniform PMBV/PVA hydrogel membrane. Subsequently, $10 \mu \mathrm{L}$ HeLa-Fucci cell suspension containing PMBV was pipetted onto the PMBV/PVA precursor hydrogel layer and allowed to flow by gravity with the dish tilted to a 45 degree angle, thus forming a thin membrane. Most of the cells were embedded in the hydrogel membrane making use of the spontaneous gelation between PMBV and PVA. The HeLa-Fucci cell-laden layer was then covered with a 1-bilayer PMBV/PVA hydrogel based on spinning-assisted LbL procedure. Finally, $2 \mathrm{~mL}$ DMEM $(10 \%$ FBS $)$ was added to each dish and all the samples were incubated at $37^{\circ} \mathrm{C}$ in a $5.0 \mathrm{vol} \% \mathrm{CO}_{2}$ humidified atmosphere.

2.4 Characterization of PMBV/PVA ultrathin hydrogel membrane

The structure and thickness of the hydrogel membranes were characterized using a Zeiss LSM 510 confocal laser-scanning microscope (CLSM; Carl Zeiss, Oberkochen, Germany). Fluorescence-labeled PMBV, poly(MPC-co-BMA-co-VPBA-co-methacryloxyethyl thiocarbamoyl rhodamine B) (PMBV-R) was used to indicate PMBV distribution in hydrogel membranes $[3,4]$.

2D

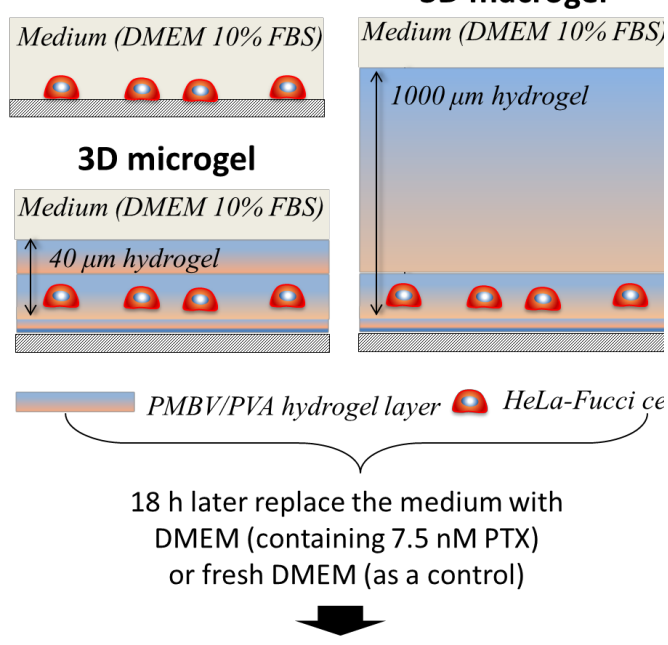

Cell cycle progression and cell viability

Fig. 2 Experimental groups

2.5 Experimental groups to study the effects of diffusional and dimensional factors

As shown in Fig. 2, three types of experiments were carried out. 3D microgel group was HeLa-Fucci cells 
encapsulated in a thin hydrogel membrane based on the procedure in section 2.3. The 3D macrogel was prepared by pipetting appropriate volumes of PMBV and PVA (for $1 \mathrm{~mm}$ height hydrogel) onto thin hydrogel membrane containing cells. HeLa-Fucci cells were also cultured on cell culture plate as a 2D group. DMEM $(10 \%$ FBS) were replaced with DMEM containing $7.5 \mathrm{nM}$ Paclitaxel (PTX) after $18 \mathrm{~h}$ in culture. Control group was also prepared by changing medium with fresh DMEM without PTX.

2.6 Cell cycle progression and cell viability analysis

The fluorescence of HeLa-Fucci cells were measured at $0,6,12,24$ and $36 \mathrm{~h}$ after stimulation using a BIOREVO BZ-9000 fluorescence microscope (KEYENCE, Osaka, Japan). Adhesive tape with a grid pattern permitting the field to be accurately and reproducibly positioned was adhered on the bottom of dishes to mark the cell distribution area. Five representative fields on each dish were selected and photographed. For quantitative analysis of fluorescent cells, the numbers of green, red, orange (both green and red), and non-fluorescent cells were determined by counting at least 400 cells in five independent fields. The percentage of green cells, corresponding to cells at the $\mathrm{S} / \mathrm{G} 2 / \mathrm{M}$ phase in the cell proliferation cycle, was calculated at each time point to generate a time-dependent curve.

Cell morphology was determined by light microscopy. In all, five different fields were randomly selected for counting at least 400 cells. The percentage of apoptotic cells was calculated for each experiment. Cells designated as apoptosis were those that displayed the characteristic morphological features of apoptosis, including cell volume shrinkage, chromatin condensation, and the presence of membrane-bound apoptotic bodies.

2.7 PTX diffusivity within the PMBV/PVA multilayer hydrogel membranes

To evaluate drug diffusion in the PMBV/PVA hydrogels, the diffusion coefficient (D) of Oregon Green ${ }^{\circledR} 488$ labeled paclitaxel (Flutax-2, P22310, Invitrogen) was determined by fluorescence correlation spectroscopy (FCS) measurements (CLSM, Carl Zeiss). Details were introduced elsewhere [4]. Briefly, the Flutax-2/PBS solution $(200 \mathrm{nM}, 2.0 \mathrm{~mL})$ was added onto PMBV/PVA hydrogels built on a $35-\mathrm{mm}$ (glass 27 Ф) glass base dish (Iwaki) and stored at $25^{\circ} \mathrm{C}$ for $24 \mathrm{~h}$ to equilibrate the samples. Then diffusion coefficients of Flutax-2 were determined at 5 random positions to get an average value. Diffusion time for the PTX to diffuse through hydrogel membranes can be calculated using equations (1).

$$
\mathrm{T}=\pi \mathrm{R}^{2} / \mathrm{D}
$$

$\mathrm{T}$ is diffusion time. $\mathrm{R}$ is thickness of the hydrogel. $\mathrm{D}$ is diffusion coefficient.

\subsection{Statistical analysis of the data}

Student's t-test was carried out to determine whether the observed differences were statistically significant ( $p$ $<0.05)$.

\section{RESULTS AND DISCUSSION}

3.1 Characterization of PMBV/PVA hydrogel membrane In the preliminary research, we investigated the dependences of the concentration of polymer solution and the spin speed of the substrate on the thickness of multilayer hydrogel. It was found that the thickness grew with increasing the concentration of PMBV (from $2.5 \%$ to $5.0 \%$ ) and PVA (from $1.0 \%$ to $5.0 \%$ ). When increasing the spin speed between 1000 and $3000 \mathrm{rpm}$, the adsorbed amount of the polymer was decreased. This further caused the decrease of thickness, which was usually observed in the spin-assisted technique [8,9]. Although uniform multilayer hydrogels could be formed at spin speed of $2000 \mathrm{rpm}$ and $3000 \mathrm{rpm}$, hydrogel layer was too thin to hold cells in the case of $3000 \mathrm{rpm}$ (data not shown). On the other side, $1000 \mathrm{rpm}$ spin speed was too low to remove all of the excess solution, which led to non-uniform distribution of polymer solution. Therefore, the optimal spin speed for our research was $2000 \mathrm{rpm}$. The optimal concentration of polymer solutions were 5.0\% PMBV and 5.0\% PVA, because hydrogel formed under this composition was strong enough to hold cells (data not shown). Fig. 3(a) clearly shows a uniform layer-by-layer structure of hydrogel membrane composed of PMBV-R and PVA with the total thickness about $40 \mu \mathrm{m}$. A probable reason for this phenomenon was that the interdiffusion behavior of polymer chains was significantly limited in the case of spinning-assisted LbL hydrogel formation, which incorporates strong centrifugal force, viscous force, and air shear force along with intermolecular forces. In particular, the viscous force induced by fast evaporation of $5.0 \%$ PVA solution and crosslinking between PMBV-R solution and PVA solution decreases the mobility and interdiffusion of PMBV-R chains. Therefore, a layer with limited distribution of PMBV was generated (Fig. 2(a) dark layer between two red layers). We have demonstrated this heterogeneous hydrogel structure was stable in cell culture medium and viability of the cells in the hydrogel layer was high $(>90 \%)[3,4]$. The cells could be immobilized in a $27 \mu \mathrm{m}$ monolayer (Fig. 3(b)), which makes them easy to be observed under microscopy.

\section{(a) Red part: 5.0\%PMBV-Rhodamin}

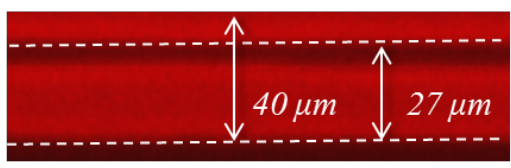

\section{(b) HeLa-Fucci cells}

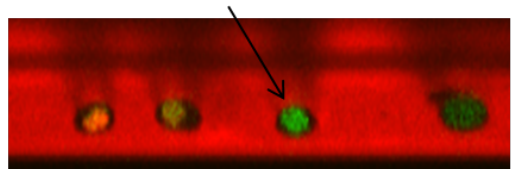

Fig. 3 Cross-sectional view of PMBV-R/PVA microgel membrane. 


\subsection{Evaluation of cell cycle progression}

As a test case, HeLa-Fucci cells were encapsulated in the multilayered PMBV/PVA hydrogel membrane for drug screening study. The PTX was chosen as a model drug, because it is a famous anticancer drug, which can block cell cycle proliferation at mitosis phase, and trigger apoptosis. Making use of Fucci technology, dynamic cell response to PTX can be easily observed without breaking up the hydrogel and cells to perform biological assays.

As shown in Fig. 4, the percentage of S/G2/M phase HeLa-Fucci cells in microgel group was higher than the control group (without stimulation) at 6 and $12 \mathrm{~h}$ after stimulation, which demonstrated PTX could induce G2/M phase block of HeLa-Fucci cells cultured in microgel membrane. However, there was no significant difference between macrogel group and control group. In other words, HeLa-Fucci cells cultured under macrogel did not show any response to stimulation, which might be explained by delayed PTX diffusion in millimeter scale hydrogel.

Further, comparison of microgel group with 2D group yielded a striking observation: HeLa-Fucci cells cultured on 2D exhibited a rapid and robust response to stimulation and the percentage of HeLa-Fucci cells in $\mathrm{S} / \mathrm{G} 2 / \mathrm{M}$ phases was much higher than microgel group at $12 \mathrm{~h}$ after stimulation. As our previous research has proved matrix dimensionality (3D confinement) can keep the cell cycle down at G1 phase [10,11], it can be speculated that there were fewer HeLa-Fucci cells of microgel group than 2D group going into $\mathrm{G} 2 / \mathrm{M}$ phase and blocked by PTX, which is a probable explanation for the different responses of microgel group compared with that of $2 \mathrm{D}$ group.

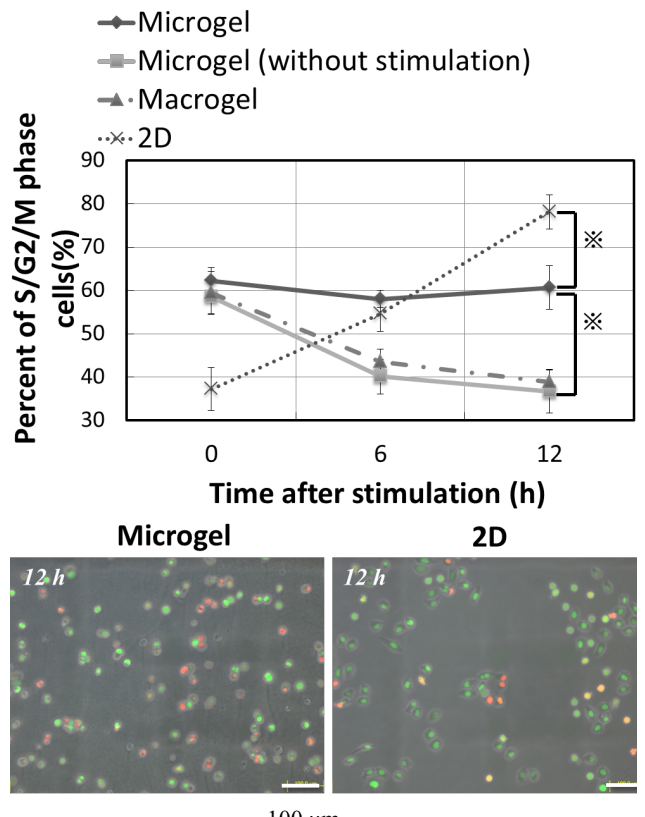

$$
100 \mu \mathrm{m}
$$

Fig. 4 Cell proliferation cycle in response to $7.5 \mathrm{nM}$ PTX stimulation in HeLa-Fucci cells. Asterisk (※) indicates a statistically significant difference $(p<0.05, n=3)$

\subsection{Evaluation of cellular viability}

As above mentioned, due to cell cycle down at G1 phase induced by $3 \mathrm{D}$ confinement, it can be thought as a factor for cancer cell to increase resistance to drugs. This mechanism could also explain why microgel group exhibited higher cell viability (15\% higher) than $2 \mathrm{D}$ group at $36 \mathrm{~h}$ after stimulation (Fig. 5).

What is more, macrogel group showed much higher cell viability $(70 \%$ higher) than microgel group. Probably, it was attributed to diffusion-dependent delay of drugs. Further, I speculated diffusion of drugs seemed to make a larger contribution to affect drug efficacy than $3 \mathrm{D}$ confinement in this study.

FCS measurement of PTX could prove this hypothesis. As diffusion coefficient of Flutax 2 was determined to be $1.3 \times 10^{-8}\left(\mathrm{~cm}^{2} / \mathrm{s}\right)$, the diffusion time for Flutax 2 to diffuse through $1 \mathrm{~mm}$ hydrogel was calculated to be 600 $\mathrm{h}$, which is much longer than our experimental time (36 h). Therefore, it is considered that long diffusion distance probably eliminates the stimulation effect of PTX.

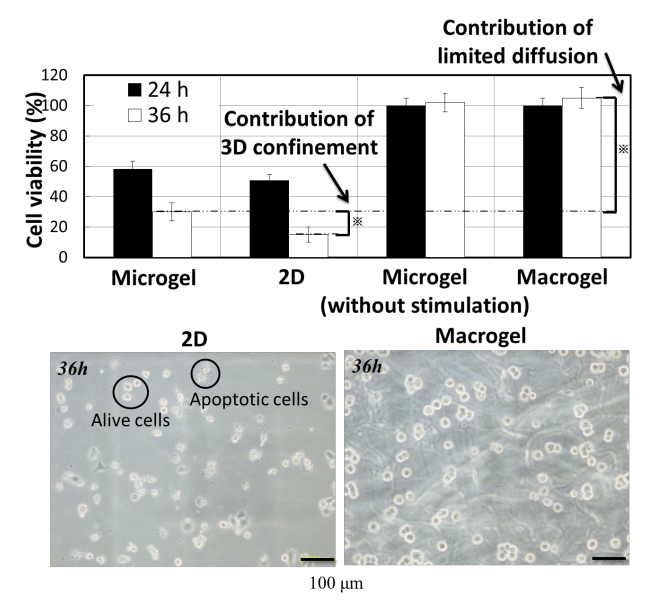

Fig. 5 Cell viability in response to $7.5 \mathrm{nM}$ PTX stimulation in HeLa-Fucci cells. Asterisk (※) indicates a statistically significant difference $(p<0.05, \mathrm{n}=3)$

\section{CONCLUTION}

The combination of the drop/coating procedure, the spinning assisted LbL technique, and PMBV and PVA that can form hydrogels spontaneously provide a versatile and experimentally convenient solution to the problem of creating a broadly useful and generalizable platform for cancer drug screening. The PMBV/PVA multilayered hydrogel membrane is a good platform to minimize barriers to the diffusion of drugs while preserving the $3 \mathrm{D}$ context. By using this microgel membrane platform and the other two platforms (2D and macrogel), PTX-induced cytotoxic action was depended on the diffusion of PTX and also matrix dimensionality. What is more, diffusion of drugs seemed to make a larger contribution. It is clear that a great deal of work remains to be done in the future. However, we believe that this work provides an insight that may prove useful in guiding the design of future drug screening systems. 


\section{Acknowledgment}

This work was supported by a Grant-in-Aid for Scientific Research on Innovative Areas" Nanomedicine Molecular Science" (No. 2306) from Ministry of Education, Culture, Sports, Science and Technology of Japan. One of the authors (B.G.) would like to acknowledge the Chinese Scholarship Council (CSC) for financial support.

\section{References}

[1] L. A. Gurski, N. J. Petrelli, X. Jia, and M. C. FarachCarson, Oncology Issues, 25, 20-25 (2010).

[2] S. Raghavan, C. J. Shen, R. A. Desai, N. J. Sniadecki, C. M. Nelson, and C. S. Chen, J. Cell Sci., 123, 2877-83 (2010).

[3] B. Gao, T. Konno, and K. Ishihara, Colloids Surf. B Biointerfaces, 108, 345-51(2013).

[4] B. Gao, T. Konno, and K. Ishihara, Biomaterials, 35, 2181-87 (2014).

[5] T. Konno and K. Ishihara, Biomaterials, 28,1770-77 (2007).

[6] K. Ishihara, T. Ueda, N. Nakabayashi, Polym. J., 23, 355-360 (1990).

[7] A. Sakaue-Sawano, H. Kurokawa, T. Morimura, A. Hanyu, H. Hama, H. Osawa, S. Kashiwagi, K. Fukami, T. Miyata, H. Miyoshi, T. Imamura, M. Ogawa, H. Masai, and A. Miyawaki, Cell, 132, 487-98 (2008).

[8] S. S. Lee, J. D. Hong, C. H. Kim, K. Kim, J. P. Koo, K. B. Lee, Macromolecules 34, 5358-60 (2001).

[9] L. L. Spangler, J. M. Torkelson, J. S. Royal, Polym. Eng. Sci. 30, 644-53 (1990).

[10] H. Oda, T. Konno and K. Ishihara, Biomaterials, 34, 5891-6 (2013).

[11] T. Aikawa, T. Konno and K. Ishihara, Soft Matter, 9, 4628-34 (2013).

(Received March 14, 2014; Accepted January 7, 2015) 